\title{
Effects of Limb Remote Postconditioning on Apoptosis and Long-Term Neurological Outcomes of Focal Cerebral Ischemia/Reperfusion Injury in Rats
}

Xianli Meng ${ }^{1}$, Yuejuan $\mathrm{Li}^{2}$, Jian Zhang ${ }^{2}$, Xiaofeng Jiang ${ }^{2}$, Xiangyang Tian $^{2}$ and Jianhua Zhao ${ }^{2 *}$

${ }^{1}$ Department of Pharmacy, Henan Provincial People's Hospital, Zhengzhou, China

${ }^{2}$ Department of Neurology, Henan Provincial People's Hospital, Zhengzhou, China

\begin{abstract}
Background and Purpose: Remote ischemic postconditioning (RPostC) is an emerging concept for cerebral infarction protection, and it has better application prospects in clinical practice. However, little is known about the neuroprotection mechanisms of RPostC in cerebral ischemia/reperfusion (I/R) injury. In the present study, we investigated the effects of RPostC on neural cells apoptosis and long-term neurological outcomes in focal cerebral $\mathrm{I} / \mathrm{R}$ injury in the rat middle cerebral artery occlusion model.
\end{abstract}

Methods: Focal cerebral ischemia was induced by middle cerebral artery occlusion using the intraluminal filament technique in male rats. RPostC was generated by 3 cycles of femoral artery 10-minute occlusion/reperfusion on the right limb at the onset of middle cerebral artery reperfusion. Adult male wistar rats were treated with remote post conditioning after 90 minutes of occlusion (beginning of reperfusion). Infarct volumes were assessed at $24 \mathrm{~h}$ and $21 \mathrm{~d}$ of stroke onset. Neurological scores were assessed at $24 \mathrm{~h}$ and $3 \mathrm{~d}, 5 \mathrm{~d}, 7 \mathrm{~d}, 10 \mathrm{~d}, 14,21 \mathrm{~d}$ after the onset of middle cerebral artery reperfusion. Apoptosis-related molecules were studied at $24 \mathrm{~h}$ of stroke onset by Western blotting.

Results: RPostC treatment up-regulated Bcl-2 and heat-shock protein 70 (HSP70) expression and downregulated Bax expression. RPostC treatment also reduced infarct volumes at $24 \mathrm{~h}$ and $21 \mathrm{~d}$, meanwhile, it also improved the neurologic scores and the long-term neurological outcomes compared with the I/R-only group.

Conclusion: These findings indicate that RPostC inhibits focal cerebral I/R injury and improves the neurological outcomes. This neuroprotective effect is likely achieved by anti-apoptotic mechanisms.

Keywords: Remote ischemic postconditioning; Ischemia/reperfusion; Apoptosis; Middle cerebral artery occlusion model; Heat-shock protein 70; Bcl-2; Bax

\section{Introduction}

Brain ischemia is becoming a leading cause of morbidity and mortality world-wide [1]. Its major pathophysiological manifestation is an acute brain ischemia-reperfusion injury [2]. Despite extensive researchs on reperfusion injury treatment in the past several decades, few neuroprotectants have been successfully from basic research into clinical application. A variety of clinical trials of pharmacological neuroprotective strategies in stroke have been disappointing [3-5]. Therefore, innovative treatment strategies for protecting brain against the detrimental effects of this form of injury are required in order to improve clinical outcomes in patients with brain ischemic injury. Researchers are now interested in the brain's endogenous strategies for neuroprotection [6,7]. Harnessing the endogenous protection elicited by the brain's ability to "condition" itself has recently emerged as a powerful new strategy for limiting brain injury $[8,9]$.

Ischemic postconditioning (PostC), which is defined as rapid intermittent interruptions of blood flow in the early phase of reperfusion and mechanically alters the hydrodynamics of reperfusion, has been verified to protect tissues from I/R injury [10]. The clinical applicability of PostC is limited because PostC can interrupt intermittent reperfusion of vital organs and require mechanical intervention, which is improbable in acute coronary syndromes and stroke [11]. The concept of ischemic PostC has been extended to remote ischemic postconditioning (RPostC), which induced by cycles of a few minutes of $\mathrm{I} / \mathrm{R}$ applied to a distal artery territory (femoral or renal artery) either immediately after the beginning of heart reperfusion or just a few minutes before heart reperfusion [11-13].
RPostC has greater potential for clinical application than conventional ischemic PostC, because it cannot only be performed following ischemia, but also protects ischemia-sensitive organs through measures on the non-critical remote organs, avoiding the high risk of inducing ischemia from PostC in the vital organ, such as the brain or the heart [14]. RPostC is a simple and harmless method that provides a new tool to protect organs from $\mathrm{I} / \mathrm{R}$ injury. It is a kind of endogenous protective measure, which can trigger an endogenous protective effect, enhance ischemic tolerance in the brain, and ultimately reduce nervous system damage following ischemia-reperfusion [15]. Recently, the protective effect of RPostC against focal cerebral ischemia in rats was also verified [16]. However, its potential protective mechanisms have not been established.

Recent developments in cardiac physiology have indicated that PostC significantly reduces infarct size and inhibits inflammation and apoptosis [17-21]. Zhao [22] at first documented that PostC reduced infarct size in cerebral I/R injury. However, the effects of RPostC on long-term neurogocal outcomes and whether the protective effect of it is

*Corresponding author: Jianhua Zhao, the Department of Neurology, Henan Provincial People's Hospital, NO.7 Weiwu Road, 450003 Zhengzhou, China, Tel: 13937103138; E-mail: Sjnk2011@163.com

Received November 24, 2015; Accepted August 25, 2015; Published August 25,2015

Citation: Meng X, Yuejuan Li, Zhang J, Jiang X, Tian X, et al. (2015) Effects of Limb Remote Postconditioning on Apoptosis and Long-Term Neurological Outcomes of Focal Cerebral Ischemia/Reperfusion Injury in Rats. J Diabetes Metab 6: 596. doi:10.4172/2155-6156.1000596

Copyright: (c) 2015 Meng X, et al. This is an open-access article distributed under the terms of the Creative Commons Attribution License, which permits unrestricted use, distribution, and reproduction in any medium, provided the original author and source are credited. 
associated with inhibition of apoptosis in-vivo reported less previously. In the current study, we tested the role of RPostC in apoptosis focal cerebral I/R injury in rats

\section{Materials and Methods}

\section{Animal models of focal cerebral ischemia}

The animals were randomly divided into three groups of experiments in sham, I/R and RPostC groups. All treatments and outcome measurements were performed by investigators blinded to the surgical groups. All experiments were performed following an institutionally approved protocol in accordance with the National Institutes of Health Guide for the Care and Use of Laboratory Animals. Male Wistar rats (280 to $330 \mathrm{~g}$ ) were used. Animals were anesthetized with $1 \%$ to $2 \%$ isoflurane under spontaneous respiration in a $30 \%$ oxygen $/ 70 \%$ nitrous oxide mixture and subjected to MCAO as described previously, with minor modifications [23]. In brief, we exposed the right common carotid artery, internal carotid artery, and external carotid artery surgically. A 4-0 monofilament nylon suture with a rounded tip was inserted into the internal carotid artery through the external carotid artery stump and gently advanced to occlude the MCA. After 90 minutes of MCAO, the suture was removed to restore blood flow (reperfusion confirmed by laser Doppler). Sham-operated rats were manipulated in the same way, but the MCA was not occluded. Regional cerebral blood flow ( $\mathrm{rCBF}$ ) was monitored by laser-Doppler flowmeter with using of a flexible probe over the skull as described earlier [24]. rCBF was measured before ischemia, during MCAO, and during reperfusion. Animals that did not show a CBF reduction of at least $70 \%$ were excluded from the experimental group, as well as animals that died after ischemia induction. Mean arterial blood pressure (of the left femoral artery), $\mathrm{pH}$, arterial blood gases, and blood glucose levels before, during, and after ischemia were measured. All surgical procedures were performed under an operating stereomicroscope.

Rectal temperature was maintained between $37^{\circ} \mathrm{C}$ and $37.5^{\circ} \mathrm{C}$ with a thermostat-controlled heating pad. The right femoral artery was cannulated, and physiologic parameters including rectal temperature, mean arterial blood pressure (MABP), $\mathrm{pH}, \mathrm{PCO} 2$, and $\mathrm{PO} 2$ were monitored throughout all experiments.

\section{Remote ischemic postconditioning (RPostC)}

An incision along the vascular route was made on each side of the femoral triangle of the hind-limb, and the bilateral femoral arteries were dissected out. The left femoral artery was cannulated for continuous monitoring of arterial blood pressure, and blood samples were analyzed for arterial blood gases and plasma glucose before the beginning of MCAO, at the onset of reperfusion and 90 minutes after reperfusion.

RPostC was induced by clamping the right femoral arteries for 3 cycles of 10 -minute $\mathrm{I} / \mathrm{R}$ with an interval of 10 minutes at the onset of MCA reperfusion. A rectal probe was inserted to monitor core temperature. The animal was irradiated with a heating lamp to maintain the rectal temperature at about $37^{\circ} \mathrm{C}$. The wounds were sutured after RPostC. Sham RPostC operations and all surgical procedures, except right femoral artery occlusion, used anesthesia.

\section{Analysis of infarct volumes}

For brain infarction at $24 \mathrm{~h}$ after ischemia, rats were killed with a lethal dose of sodium pentobarbital and transcardially perfused to remove all intravascular blood. Coronal brain sections (2mm thick) were stained with 2.3.5-triphenyltetrazolium chloride (TTC, Sigma, St
Louis, MO, USA). Infarct volumes were quantified using computerassisted image analysis [22]. To eliminate confounding effects of edema and swelling, the indirect method was used (contralateral volume minus uninfarcted ipsilateral volume). For $21 \mathrm{~d}$ brain infarction, frozen sections (interval $2 \mathrm{~mm}$ ) were stained with hematoxylin and eosin (HE). Infarction volumes were quantified as described earlier [23] and expressed as percentage of hemisphere.

\section{Assessments of sensorimotor functions}

An 18-point Modified neurological severity score (mNSS) and adhesive tap-removal test were used for the assessment of posttraumatic neurological impairments, as previous described [25-27]. All outcome measurements were performed by investigators blinded to the surgical groups. The mNSS and adhesive tap-removal test were assessed at $1 \mathrm{~d}$, $3 \mathrm{~d}, 5 \mathrm{~d}, 7 \mathrm{~d}, 10 \mathrm{~d}, 14 \mathrm{~d}$ and $21 \mathrm{~d}$ after experiments by following standard methods. All rats had been pretested before 5 days of experiments and trained for 3 days to eliminate the individual difference rats. Adhesive tap-removal test was assessed for sensorimotor neurological deficits, and performed in triplicate and an average value calculated for each rat on each day of testing. Baseline assessment was done the day before MCAO.

\section{Western blotting}

Western blotting analysis was performed by following the standard method as we described previously [28]. Briefly, brain hemisphere tissues from the right cortical samples of rats were dissected on ice followed by protein extraction. Protein concentrations in the supernatants were measured with Bradford assay (Bio-Rad, Hercules, CA, USA). Equal amount of protein were separated in a $4-20 \%$ Trisglycinegel (Invitrogen) $(40 \mu \mathrm{g} / \mathrm{lane})$ and then transferred onto PVDF Membranes. The membranes were incubated with primary anti-HSP70, anti-Bax and anti-Bcl-2 antibody (1:1000; Biovendor R\&D, NC, USA) and anti- $\beta$-actin antibody (1:10000; Sigma) at $4{ }^{\circ} \mathrm{C}$ overnight. And then the membranes were incubated with an anti-rabbit secondary antibody. (1:10000, GE Healthcare, UK) for $1 \mathrm{~h}$ at room temperature, developed by enhanced chemiluminescent (Pierce, Rockford, IL, USA). Densitometric analysis was performed for quantitation with Imagin software.

\section{TUNEL assay}

A TUNEL assay was used to assess DNA damage. The sections from the right cortical samples ( $\mathrm{n}=5$ for each group) were treated as instructed with an in situ cell death detection kit (Roche, Basel, Switzerland). Diaminobenzidine (Sigma) was used as a chromogen. TUNEL-positive cells displayed brown staining within the nucleus of apoptotic cells. DNA fragmentation was quantified under high-power magnification $(\times 400)$ by an investigator who was blinded to the studies and was expressed as number per square millimeter.

\section{Statistical analysis}

The statistical analyses were performed by using SPSS 17.0 for Windows. Data were expressed as mean+s.e.m. Infarction volume was analyzed by Student $t$ test. Mortality rate was analyzed by 2 -sided Fisher exact test. Neurobehavioral assessments were analyzed by repeated measures ANOVA. Differences with $\mathrm{P}<0.05$ were considered statistically significant.

\section{Results}

\section{Neurological outcomes and mortality rate}

To test whether the RPostC can improve neurological outcomes in 
longer survival time after stroke, 16 rats ( $\mathrm{n}=8$ per group) were treated with either I/R or RPostC. There was a statistically significant in RPostC group compared with $\mathrm{I} / \mathrm{R}$ group on modified neurological severity score (mNSS) $(\mathrm{P}=0.001)$ and adhesive tap-removal test $(\mathrm{P}=0.000)$. There was a significant interaction effect between treatments and time for neurological functional recovery (up to 21 days after focal stroke in rats) (Figure 1A). However, there was no significant difference in mortality between $\mathrm{RPostC}$ group $(5 / 20,25 \%)$ and I/R group $(8 / 20$, $40 \% ; \mathrm{P}=0.087$ ) (Figure 1B).

\section{$\mathrm{RPostC}$ protected against focal ischemia and reperfusion}

At $24 \mathrm{~h}$ after ischemia, infarct volume in RPostC group was significantly smaller than I/R group $(P=0.031)$, stained by TTC. Representative samples of TTC-stained brain sections in the sham, $\mathrm{I} / \mathrm{R}$ and RPostC groups are shown in Figure 2A. The infarct volume of 3 groups in sham, I/R and RPostC groups are shown in Figure 2B. At $21 \mathrm{~d}$ after stroke, frozen sections (interval $2 \mathrm{~mm}$ ) were stained with hematoxylin and eosin (HE) in Figure 2C. Infarction volumes were quantified as described earlier and expressed as percentage of hemisphere in Figure 2D. For the long outcomes, there was a statistically significant in RPostC group compared with I/R group with reduction rate of $9.6 \%(\mathrm{p}=0.024)$.
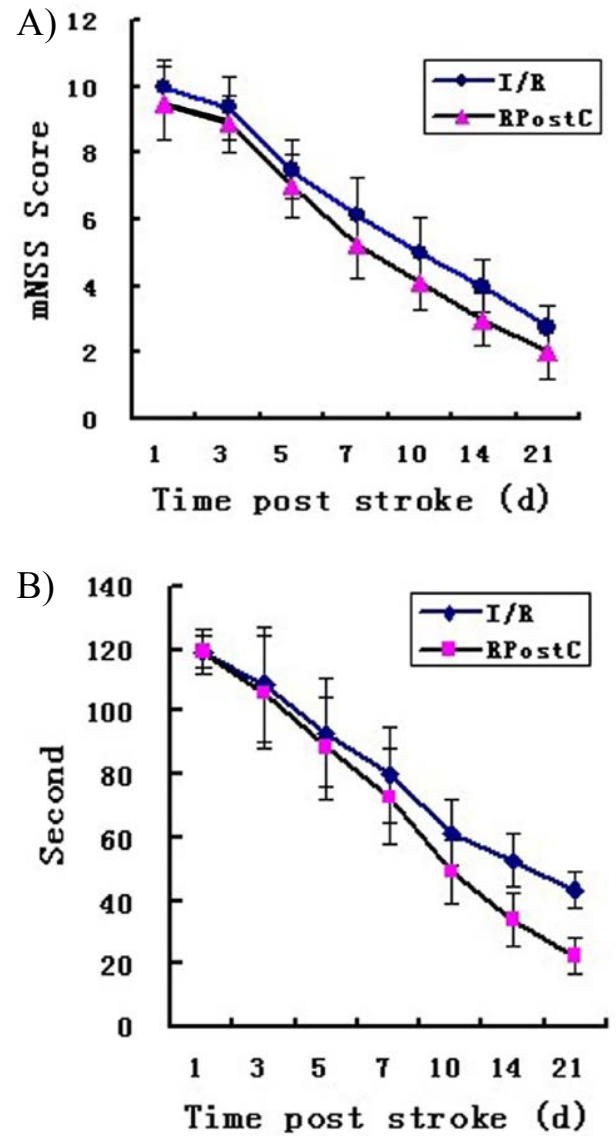

Figure 1: The neurological outcomes were assessed at 1d, 3d, 5d, 7d, $10 \mathrm{~d}, 14 \mathrm{~d}$ and $21 \mathrm{~d}$ after ischemia. Neurological function had a statistically significant better performance in RPostC group compared with I/R group. RPostC significantly decreased neurologic deficits. A) Modified neurological severity score (mNSS). B) Adhesive tap-removal test. ${ }^{*} P<0.05$ in RPostC group comparison with I/R group. (mNSS = modified neurological severity score; RPostC = remote ischemic postconditioning)
A

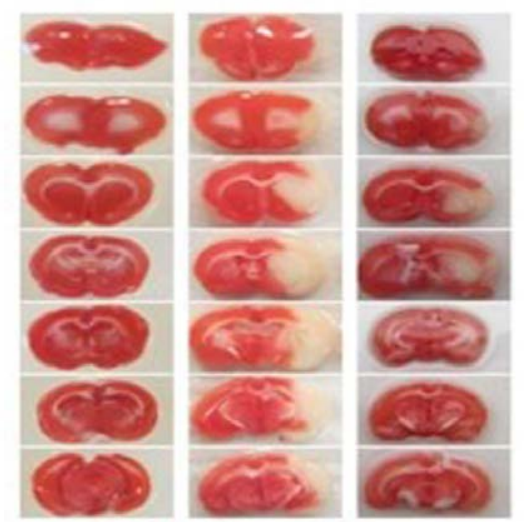

\section{Sham IR RPostC}

B
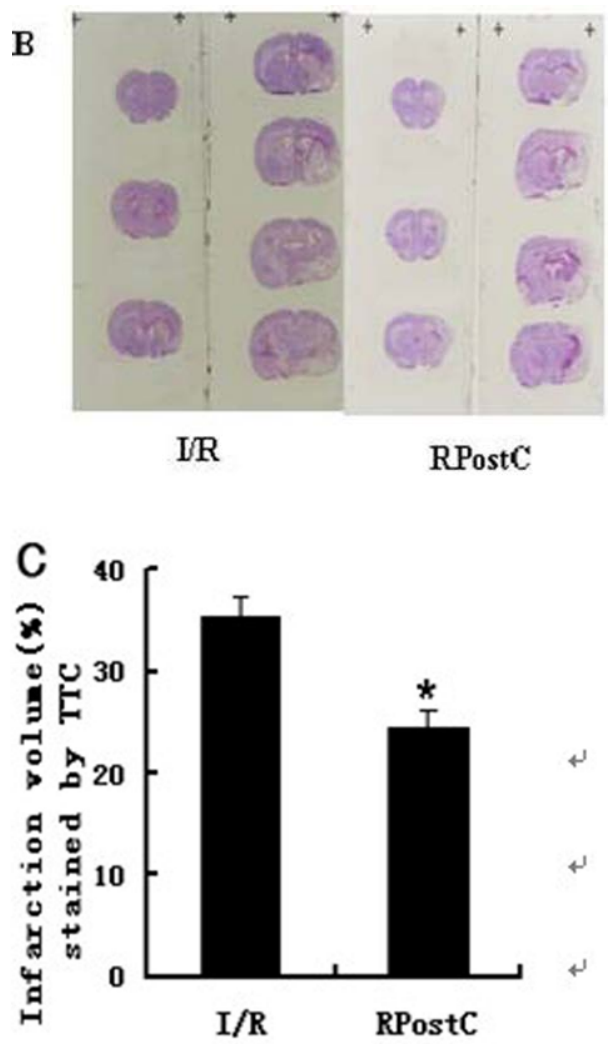

Figure 2: RPostC conferred neuroprotection against focal cerebral ischemia and reperfusion injury $(n=6)$. A) Representative TTC staining of the cerebral infarct in comparable sections of rat brain from the sham, I/R and RPostC groups at $24 \mathrm{~h}$ after reperfusion. B) Representative HE staining of the cerebral infarct in RPostC group comparable with the I/R group at $21 \mathrm{~d}$ after reperfusion. C, D) Quantification of infarct volumes, stained by TTC and HE. RPostC significantly decreased infarction volumes in comparison with the I/R group. Infarct volumes are expressed as the mean \pm s.e.m. ${ }^{*} P<0.05$ in RPostC group comparison with I/R group. (TTC $=2$, 3, 5-triphenyltetrazolium chloride; $\mathrm{HE}=$ hematoxylin and eosin; RPostC = remote ischemic postconditioning)

\section{RPostC reduced number of apoptotic cells after reperfusion}

Representative photomicrographs of TUNEL staining in the periinfarct zone of rat brain sections from sham, I/R and RPostC were 
shown in Figure 3A. TUNEL staining (brown) was negative in the brain sections of sham animals at $24 \mathrm{~h}$ after reperfusion. Figure $3 \mathrm{~B}$ : A large number of TUNEL-positive cells in the ischemic penumbra of rat brain were seen in the I/R whereas in contrast, Figure 3C: only a few TUNEL-positive cells were observed in the RPostC group. As shown in Figure 3D, RPostC significantly reduced the number of TUNELpositive cells at $24 \mathrm{~h}$ after reperfusion, in comparison with $\mathrm{I} / \mathrm{R}{ }^{\star} P<0.05$.

\section{Influence of postconditioning on apoptosis-related molecules}

Postconditioning increased the level of anti-apoptotic HSP70 and $\mathrm{Bcl}-2$ protein and inhibited Bax expression in Figures 4 and 5. The protein levels of HSP70 and Bcl-2expression in the I/R group were greater than the sham-operated rats. Moreover, this increase was much more prominent in the postconditioning group. But Bax expressions of I/R group were greater than those in sham-operated and postconditioning groups. RPostC improved HSP70 and Bcl-2 expression and decreased Bax expression.

\section{Discussion}

Cerebral I/R injuries often occur in clinical practice and are associated with frequent morbidity and mortality. Until now, the primary treatment for cerebral ischemia is recanalization of the occluded blood vessels to allow early reperfusion, either by the administration of a thrombolytic drug or intervention with mechanical devices [29]. However, it is now clear that reperfusion has the potential to induce additional lethal injuries that are not present at the end of

\section{A}
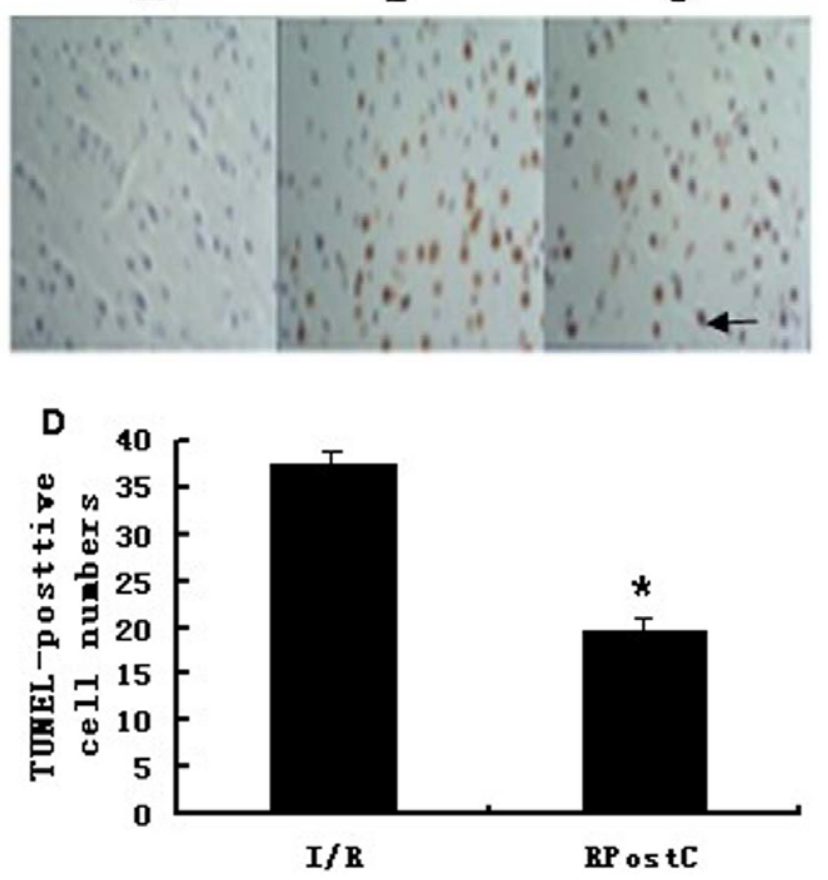

Figure 3: RPostC reduced number of apoptotic cells after reperfusion $(n=6)$. A-C) Representative photomicrographs of TUNEL staining in the peri-infarct zone of rat brain sections from sham, I/R and RPostC at $24 \mathrm{~h}$ after reperfusion. The arrow indicates TUNEL-positive cells. Scale bars $=20 \mu \mathrm{m}$. D) The bar graph shows quantitative analysis of the number of TUNEL-positive cells in the ischemic penumbra of rats in 3 groups. Values are expressed as the mean \pm s.e.m. ${ }^{*} \mathrm{P}<0.05$ in RPostC comparison with I/R. (TUNEL $=$ terminal deoxynucleotidyl transferase-mediated dUDP-biotin nick end-labeling; RPostC = remote ischemic postconditioning)

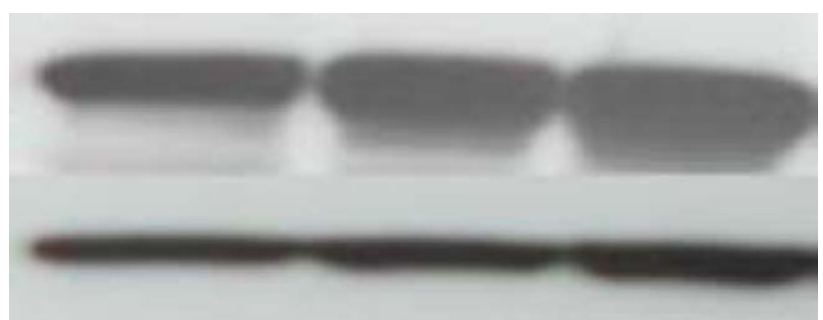

$\mathbf{A}$

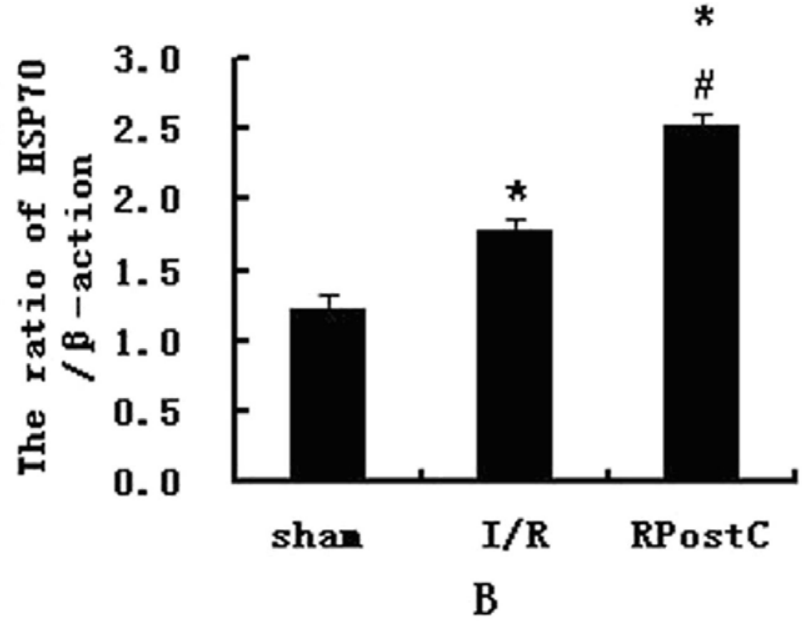

Figure 4: RPostC increased the level of HSP70 protein expression. The protein levels of HSP70 expression in the I/R group were greater than those in sham-operated rats. Moreover, this increase was much more prominent in the RPostC group. Five independent experiments yielded similar results. A) Representative blots showing the effects of RPostC on HSP70 expression. B) The bar graph showed semiquantification of the Western blotting analysis for HSP70. Values are expressed as the mean \pm s.e.m. ${ }^{*} P<0.05$ in comparison with sham. \#P $<0.05$ in RPostC comparison with $\mathrm{l} / \mathrm{R}(\mathrm{n}=5)$

the ischemic period. This phenomenon has been termed "reperfusion injury" [30]. It is important to improve the ability of brain tissue to tolerate I/R injury. Developments in brain protection have indicated that PostC is an interesting strategy to reduce reperfusion injury in multiple animal models as well as in-vitro settings [31]. But, the clinical applicability of PostC is limited because it can interrupt intermittent reperfusion of vital organs and require mechanical intervention, which is improbable in acute coronary syndromes and stroke [11].

The concept of ischemic PostC has been extended to RPostC. $\mathrm{RPostC}$ was found to induce ischemic tolerance not only within the same piece of tissue, but also in distant tissues as well as in distant organs [32]. Ren [16] further demonstrated that RPostC reduced infarct volume, significantly improved neurological function, and protected brain function in a rat model of left artery permanent occlusion. Ischemic postconditioning is shown to prevent cerebral ischemic injury in various animal experiments, so the $\mathrm{RPostC}$ has more potential contribution to the clinical treatment of stroke.

Recently, RPostC generated by 3 cycles of 15 minutes each $I / R$ of the left-hind femoral artery also protected against focal ischemia in rats [16]. The RPostC regimen was selected on the basis of its efficacy in a previously published model [33]. In the present study, our results showed that RPostC, which was induced by 3 cycles of 10 minutes I/R of the hind-limb at the onset of MCA reperfusion, reduced infarct size in the short and long term, and promoted long-term neurological 
A

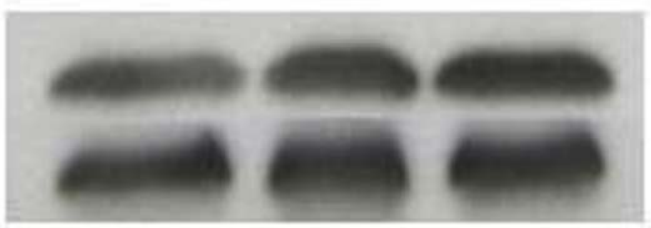

مิ
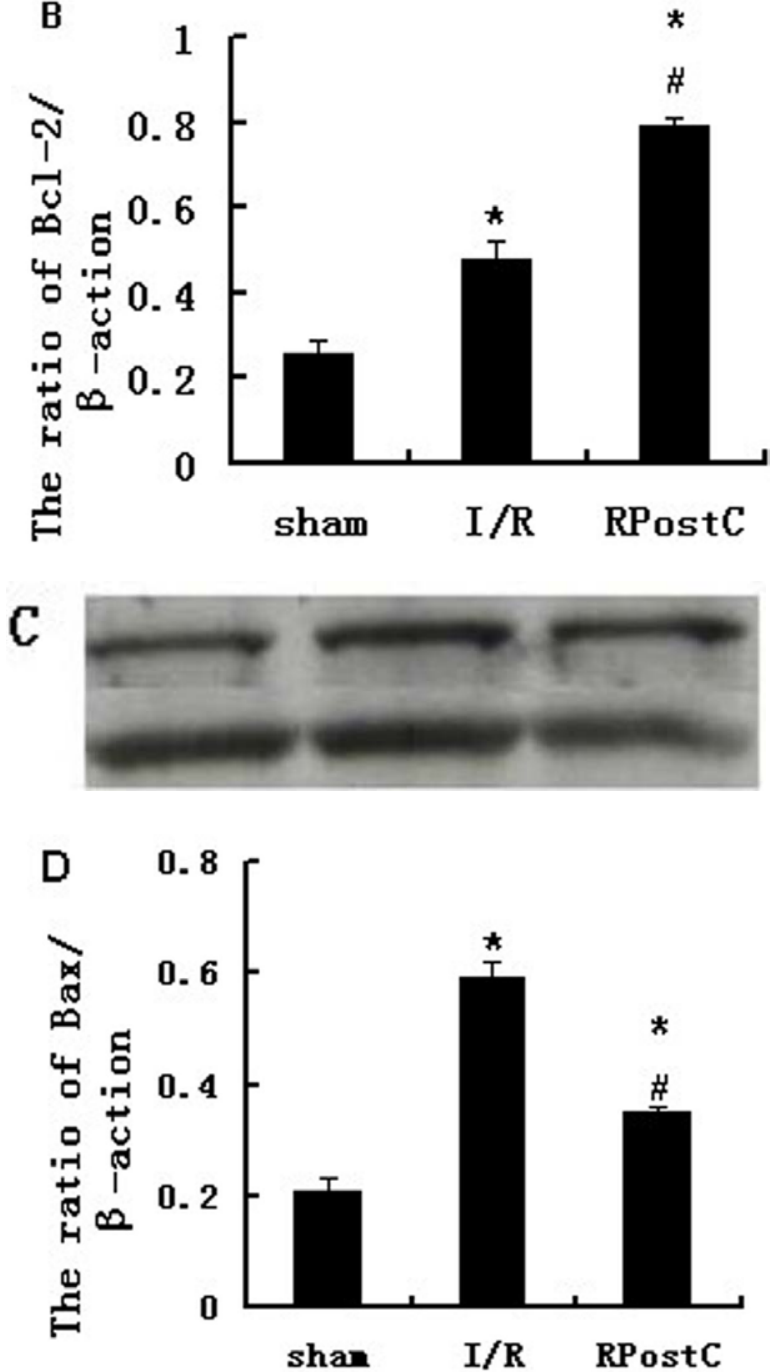

Figure 5: Bcl-2 and Bax expression at $24 \mathrm{~h}$ after stroke onset. (A), Representative blots showing the effects of RPostC on Bcl2 expression. (B) The bar graph showed semiquantification of the Western blotting analysis for Bcl-2. (C), Representative blots showing the effects of RPostC on Bax expression. (D) The bar graph showed semiquantification of the Western blotting analysis for Bax. Bars represent mean \pm s.e.m $(n=5)$. ${ }^{*} P<0.05$ in RPostC and I/R groups comparison with sham. $\# P<0.05$ in RPostC group comparison with I/R group

functional recovery after I/R injury. Our results further demonstrated that $\mathrm{RPostC}$ alleviated reperfusion injury after focal cerebral ischemia as was consistent with a previous report, and improved long-term neurological functional outcomes, which has been less reported so for.

The early moments of reperfusion are important in the pathogenesis of post-ischemic injury. A previous study has demonstrated that PostC reduces cerebral I/R injury and provides neuroprotection in contexts of both focal and global ischemia [34]. The present study further supports that opinion. However, the specific mechanisms triggered by RPostC are still unclear. In addition, the earlier study was performed after partial reperfusion in a model of permanent focal ischemia. Whether such a protective phenomenon is applicable to other I/R models need to be elucidated.

The protective mechanisms underlying RPostC have not been fully elucidated. Emerging evidence indicates that it may share common mechanistic signaling pathways with the conventional ischemic preconditioning, postconditioning, and remote preconditioning [34]. PostC has been proven to confer neuroprotection in experimental cerebral ischemia [35]. Research reported that PostC reduced neuronal death and neurobehavioral deficit after global ischemia, by inhibited apoptotic pathways [36,37]. And, studies have demonstrated that PostC exerts an anti-apoptotic effect on the heart and cerebral both in-vivo and in-vitro $[38,39]$.

Previous work has shown that PostC can increase HSP70 expression, which is associated with a reduction of cytochrome $\mathrm{c}$ release from the mitochondria [39,40]. Ischemic injury has been shown to induce the expression of heat shock proteins (HSPs). HSPs are a family of functionally-related proteins and are believed to be involved in the cellular repair processes by re-folding proteins and to act as molecular chaperones in physiological processes [41]. The highly inducible member of the family of HSPs, HSP70 has been found to be associated with tolerance to ischemic injury in the brain [42]. Just as PostC, Our study also revealed that RPostC increased the level of HSP70 in the cortex during cerebral I/R. Whether other endogenous protective molecules besides HSP70 are involved in the protection afforded by RPostC in cerebral ischemia requires further study.

In addition, our study showed that RPostC significantly inhibited apoptosis of cortical neurons caused by I/R injury, which was proved by DNA fragmentation and activated caspase-3. To further clarify the mechanism of RPostC protection, we investigated the expressions of key apoptosis-related molecules, including Bcl-2 and Bax. Our study showed that RPostC increased the level of anti-apoptotic $\mathrm{Bcl}-2$ protein and inhibited Bax expression.

In conclusion, our study demonstrated that RPostC reduced cerebral infarct size in the short and long term, and improved neurological outcomes in the long term after I/R injury. This neuroprotective effect was associated with inhibiting apoptosis molecules of the mitochondrial pathway and activating endogenous protective protein.

\section{Sources of Funding and Support}

This project was supported in part by a grant from the Health Bureau of Henan Province, China (No.201003111), and Science and Technology of Henan province, China (No.132300410425).

\section{Conflict of Interest}

The authors declare that there is no conflict of interest with any financial organization or corporation or individual that can inappropriately influence this work.

\section{Disclosures}

\section{None}

\section{Acknowledgement}

We express gratitude to the central laboratory of Henan Provincial People's Hospital for providing the resources. 
Citation: Meng X, Yuejuan Li, Zhang J, Jiang X, Tian X, et al. (2015) Effects of Limb Remote Postconditioning on Apoptosis and Long-Term Neurological Outcomes of Focal Cerebral Ischemia/Reperfusion Injury in Rats. J Diabetes Metab 6: 596. doi:10.4172/2155-6156.1000596

Page 6 of 6

\section{References}

1. Wardlaw JM, von Kummer R, Farrall AJ, Chappell FM, Hill M, et al. (2010) A large web-based observer reliability study of early ischaemic signs on computed tomography. The Acute Cerebral CT Evaluation of Stroke Study (ACCESS). PLoS One 5: e15757.

2. Sinanovi O (2010) Neuropsychology of acute stroke. Psychiatr Danub 22 278-281

3. Lakhan SE, Pamplona F (2012) Application of mild therapeutic hypothermia on stroke: a systematic review and meta-analysis. Stroke Res Treat 2012 295906.

4. Mantz J, Degos V, Laigle C (2010) Recent advances in pharmacologic neuroprotection. Eur J Anaesthesiol 27: 6-10.

5. Chavez JC, Hurko O, Barone FC, Feuerstein GZ (2009) Pharmacologic interventions for stroke: looking beyond the thrombolysis time window into the penumbra with biomarkers, not a stopwatch. Stroke 40: e558-563.

6. Durukan A, Tatlisumak T (2010) Preconditioning-induced ischemic tolerance: a window into endogenous gearing for cerebroprotection. Exp Transl Stroke Med 2: 2.

7. Bhuiyan MI, Kim YJ (2010) Mechanisms and prospects of ischemic tolerance induced by cerebral preconditioning. Int Neurourol J 14: 203-212.

8. Lehotský J, Burda J, Danielisová V, Gottlieb M, Kaplán P, et al. (2009) Ischemic tolerance: the mechanisms of neuroprotective strategy. Anat Rec (Hoboken) 292: 2002-2012.

9. Ferrara A, El Bejaoui S, Seyen S, Tirelli E, Plumier JC (2009) The usefulness of operant conditioning procedures to assess long-lasting deficits following transient focal ischemia in mice. Behav. Brain Res 205: 525-34.

10. Zhao H (2011) The Protective Effects of Ischemic Postconditioning against Stroke: From Rapid to Delayed and Remote Postconditioning. Open Drug Discov J 5: 138-147.

11. Liu Q, Zhou S, Wang $Y$, Qi F, Song $Y$, et al. (2014) A feasible strategy for focal cerebral ischemia-reperfusion injury: remote ischemic postconditioning Neural Regen Res 9: 1460-1463.

12. Andreka G, Vertesaljai M, Szantho G, Font G, Piroth Z, et al. (2007) Remote ischaemic postconditioning protects the heart during acute myocardial infarction in pigs. Heart 93: 749-752

13. Szijártó A, Czigány Z, Turóczi Z, Harsányi L (2012) Remote ischemic perconditioning--a simple, low-risk method to decrease ischemic reperfusion injury: models, protocols and mechanistic background. A review. J Surg Res 178: 797-806.

14. Ren C, Gao M, Dornbos D 3rd, Ding Y, Zeng X, et al. (2011) Remote ischemic post-conditioning reduced brain damage in experimental ischemia/ reperfusion injury. Neurol Res 33: 514-519.

15. Pignataro G, Cuomo O, Vinciguerra A, Sirabella R, Esposito E, et al. (2013) NCX as a key player in the neuroprotection exerted by ischemic preconditioning and postconditioning. Adv Exp Med Biol 961: 223-240.

16. Ren C, Yan Z, Wei D, Gao X, Chen X, et al. (2009) Limb remote ischemic postconditioning protects against focal ischemia in rats. Brain Res 1288: 88-94.

17. Li WN, Wu N, Shu WQ, Guan YE, Jia DL (2014) The protective effect of fasudil pretreatment combined with ischemia postconditioning on myocardial ischemia/reperfusion injury in rats. Eur Rev Med Pharmacol Sci 18: 2748-2758.

18. Zhang GM, Wang Y, Li TD, Li XY, Su SP, et al. (2014) Post-conditioning with gradually increased reperfusion provides better cardioprotection in rats. World J Emerg Med 5: 128-134

19. Thuny F, Lairez O, Roubille F, Mewton N, Rioufol G, et al. (2012) Postconditioning reduces infarct size and edema in patients with ST-segment elevation myocardial infarction. J Am Coll Cardiol 59: 2175-2181.

20. Sanada S, Komuro I, Kitakaze M (2011) Pathophysiology of myocardial reperfusion injury: preconditioning, postconditioning, and translational aspects of protective measures. Am J Physiol Heart Circ Physiol 301: H1723-1741.

21. Sun HY, Wang NP, Halkos M, Kerendi F, Kin H, et al. (2006) Postconditioning attenuates cardiomyocyte apoptosis via inhibition of JNK and p38 mitogenactivated protein kinase signaling pathways. Apoptosis 1: 1583-93.

22. Zhao H, Sapolsky RM, Steinberg GK (2006) Interrupting reperfusion as a stroke therapy: ischemic postconditioning reduces infarct size after focal ischemia in rats. J Cereb Blood Flow Metab 26: 1114-1121.

23. Asahi M, Asahi K, Wang X, Lo EH (2000) Reduction of tissue plasminogen activator-induced hemorrhage and brain injury by free radical spin trapping after embolic focal cerebral ischemia in rats. J Cereb Blood Flow Metab 20: 452-457.

24. Zhao BQ, Wang S, Kim HY, Storrie H, Rosen BR, et al. (2006) Role of matrix metalloproteinases in delayed cortical responses after stroke. Nat Med 12 441-445.

25. Tsenter J, Beni-Adani L, Assaf $Y$, Alexandrovich AG, Trembovler V, et al. (2008) Dynamic changes in the recovery after traumatic brain injury in mice: effect of injury severity on T2-weighted MRI abnormalities, and motor and cognitive functions. J Neurotrauma 25: 324-333.

26. Beni-Adani L, Gozes I, Cohen Y, Assaf Y, Steingart RA, et al. (2001) A peptide derived from activity-dependent neuroprotective protein (ADNP) ameliorates injury response in closed head injury in mice. J Pharmacol Exp Ther 296: 57-63.

27. Bermpohl D, You Z, Korsmeyer SJ, Moskowitz MA, Whalen MJ (2006) Traumatic brain injury in mice deficient in Bid: effects on histopathology and functional outcome. J Cereb Blood Flow Metab 26: 625-633.

28. Wang X, Liu J, Zhu H, Tejima E, Tsuji K, et al. (2008) Effects of neuroglobin overexpression on acute brain injury and long-term outcomes after focal cerebral ischemia. Stroke 39: 1869-1874.

29. Stead LG, Gilmore RM, Bellolio MF, Rabinstein AA, Decker WW (2008) Percutaneous clot removal devices in acute ischemic stroke: a systematic review and meta-analysis. Arch Neurol 65: 1024-1030.

30. Aronowski J, Strong R, Grotta JC (1997) Reperfusion injury: demonstration of brain damage produced by reperfusion after transient focal ischemia in rats. J Cereb Blood Flow Metab 17: 1048-1056.

31. Pignataro G, Meller R, Inoue K, Ordonez AN, Ashley MD, et al. (2008) In vivo and in vitro characterization of a novel neuroprotective strategy for stroke: ischemic postconditioning. J Cereb Blood Flow Metab 28: 232-241.

32. Tsubota H, Marui A, Esaki J, Bir SC, Ikeda T, et al. (2010) Remote postconditioning may attenuate ischaemia-reperfusion injury in the murine hindlimb through adenosine receptor activation. Eur J Vasc Endovasc Surg 40: $804-809$.

33. Zhou Y, Fathali N, Lekic T, Ostrowski RP, Chen C, et al. (2011) Remote limb ischemic postconditioning protects against neonatal hypoxic-ischemic brain injury in rat pups by the opioid receptor/Akt pathway. Stroke 42: 439-444.

34. MaXD, Song JN, Zhang M, An JY, Zhao YL, et al. (2015) Advances in research of the neuroprotective mechanisms of cerebral ischemic postconditioning. Int J Neurosci 125: 161-169.

35. Zhao H (2009) Ischemic postconditioning as a novel avenue to protect against brain injury after stroke. J Cereb Blood Flow Metab 29: 873-885

36. Wang JY, Shen J, Gao Q, Ye ZG, Yang SY, et al. (2008) Ischemic postconditioning protects against global cerebral ischemia/reperfusioninduced injury in rats. Stroke 39: 983-990.

37. Prasad SS, Russell M, Nowakowska M (2011) Neuroprotection induced in vitro by ischemic preconditioning and postconditioning: modulation of apoptosis and PI3K-Akt pathways. J Mol Neurosci 43: 428-442.

38. Wu QL, Shen T, Shao LL, Ma H, Wang JK (2012) Ischemic postconditioning mediates cardioprotection via PI3K/GSK-3 $\hat{I}^{2} / \hat{I}^{2}$-catenin signaling pathway in ischemic rat myocardium. Shock 38: 165-169.

39. Zhao JH, Meng XL, Zhang J, Li YL, Li YJ, et al. (2014) Oxygen glucose deprivation post-conditioning protects cortical neurons against oxygenglucose deprivation injury: role of HSP70 and inhibition of apoptosis. Huazhong Univ Sci Technolog Med Sci 34: 18-22.

40. Ouyang YB, Xu LJ, Sun YJ, Giffard RG (2006) Overexpression of inducible heat shock protein 70 and its mutants in astrocytes is associated with maintenance of mitochondrial physiology during glucose deprivation stress. Cell Stress Chaperones 11: 180-186.

41. Henderson B (2010) Integrating the cell stress response: a new view of molecular chaperones as immunological and physiological homeostatic regulators. Cell Biochem Funct 28: 1-14.

42. da Silva KP, Borges JC (2011) The Molecular Chaperone Hsp70 Family Members Function by a Bidirectional Heterotrophic Allosteric Mechanism. Protein \& Peptide Letters 18: 132-142. 